\title{
Research Article \\ Optical Conduction Resonance in Self-Assembled Metal Nanoparticle Array-Dielectric Thin Films
}

\author{
Liangmin Zhang (D) \\ Department of Physics, Physical Sciences, and Geology, California State University-Stanislaus, Turlock, CA 95382, USA \\ Correspondence should be addressed to Liangmin Zhang; lzhang@csustan.edu
}

Received 31 May 2018; Revised 21 September 2018; Accepted 14 November 2018; Published 10 December 2018

Academic Editor: Yogendra Mishra

Copyright @ 2018 Liangmin Zhang. This is an open access article distributed under the Creative Commons Attribution License, which permits unrestricted use, distribution, and reproduction in any medium, provided the original work is properly cited.

\begin{abstract}
Optical conduction resonance- (OCR-) enhanced third-order optical nonlinearity of two dimensional (2D) periodic gold nanoparticle array-dielectric thin films has been investigated. The third-order optical susceptibility of periodic gold nanoparticle array embedded in silica thin film shows $\sim 10^{4}$ enhancement comparing to gold nanoparticle colloids. The $2 \mathrm{D}$ gold nanoparticle arrays were synthesized by using the electrostatic self-assembly (ESA) technique. During the fabrication process, the positively or negatively functionalized gold nanoparticles are automatically self-aligned to establish a $2 \mathrm{D}$ array with a very small interparticle spacing due to the polymer shell on the metal particles. Then, a monolayer of silica can be coated on the top of the 2D metal nanoparticle array. This type of $2 \mathrm{D}$ gold nanoparticle array-dielectric thin films has high volume fraction of gold nanoparticles. According to the extended Maxwell-Garnett theory, this kind of films can exhibit OCR. The OCR frequency can be tuned from visible to mid-infrared by controlling the gold nanoparticle volume fraction. During OCR, the real part of the composite dielectric constant is zero to make the induced electromagnetic waves in gold nanoparticles to couple effectively within the film. The open-aperture z-scan technique is used to measure the nonlinear optical properties of the ESA films.
\end{abstract}

\section{Introduction}

Gold $(\mathrm{Au})$ nanoparticles and clusters are known to possess a fast and extremely large nonlinear optical susceptibility (third-order susceptibility of $\chi^{(3)}$ can be as much as $10^{6}$ times larger than that of the standard reference of $\mathrm{CS}_{2}$ ) $[1,2]$. Although linear optical properties of small metal particles and metal colloids have been studied for at least a century based on Mie and Maxwell-Garnett (MG) theories [3-5], because pure metal thin films have large optical attenuation constants and do not normally transmit optical radiation, embedding metal nanoparticles in a dielectric matrix to provide a smart way to investigate the nonlinear optical properties is currently an intensive research area. The experimental investigation of nonlinear optical phenomena in $\mathrm{Au}$ nanoparticle-incorporated systems started in the 1980s [6-8], most of the research work is concentrated on low metal particle volume fraction. After Richard and coworkers performed the first measurement of the nonlinear optical response of metal colloids in 1985 [6], subsequently, in the last three decades, numerous other research work has contributed to our understanding of such composites, in which the concentration of metal particles is usually very low $\left(\sim 10^{-6}-10^{-5}\right.$ in volume fraction). To my knowledge, only quite a small volume of research has been directed toward the composites with high volume fraction of metal nanoparticles [9-11]. In particular, few experimental investigations concentrated on the OCR-enhanced nonlinear optical susceptibilities have been performed. After the year 2000, giant enhancement (up to $10^{10}-10^{15}$ ) of optical susceptibility in metal nanocomposites consisting of metal nanoparticles and dielectrics has been reported [12-16]. This has led to a resurgence of activities both in the design of plasmon resonance-based materials and the understanding of fundamental mechanisms [17-25]. Although some questions on the exact origin of the enhanced nonlinear optical susceptibility still remain $[20,26]$, it is generally agreed that closely ordered periodic metal nanostructures and aggregated nanometal clusters can provide a large local-field enhancement because of small interparticle spacing and collective oscillation of conduction electron gas [12-15] that is called OCR. The OCR phenomenon in high-volume Au particle systems 
has been theoretically predicted in the 1970s [27, 28]. Microscopically, when light is incident on the system, the free electrons in metal nanoparticles are driven by a periodic field. The free electrons respond to the periodic local field, sloshing back and forth within the volume of the nanoparticles. This is actually a periodic conduction current within the particles causing a periodic polarization field around the particles-both periods being equal to that of the incident radiation. At a certain frequency the phases of the conduction currents in all the particles become nearly equal, resulting in a strong oscillation field, which enhances the local field. The collective resonance of photoconductivity occurs at this optical frequency. This resonance is not the normal free-electron plasmon resonance [27, 28]. Instead, at the OCR frequencies, the photoconductivity of the system is maximized. Only at a very low concentration of metal nanoparticles, the OCR and surface plasmon resonance (SPR) occur at the same frequency $[27,28]$. As far as I know, the ion implantation laser ablation [29], electron beam deposition [30], and sputtering techniques are commonly used to fabricate metal nanoparticle-dielectric thin films with high volume fraction of metal nanoparticles. This work reports using the electrostatic self-assembly (ESA) technique to fabricate two-dimensional (2D) Au nanoparticle arraydielectric thin films containing high volume fraction of $\mathrm{Au}$ nanoparticles to investigate this type of resonance through theoretical calculations and experimental characterizations. In the theory section, I will first review some fundamental theoretical work and show the computed results based on the fundamental work. In the sample preparation section, I will briefly present the sample fabrication process. In the characterization section, the measured results of third-order susceptibility from the thin films containing high-volume Au particles will be shown.

\section{Theory}

In the 1970s, Marton and Lemon developed the MG theory to explain several resonance features in aggregated metal systems and metal-dielectric thin films with high volume fraction of metal nanoparticles $[27,28]$. In addition to bulk plasmon and SPRs, this model predicts that there is OCR in the system with a high volume fraction of metal nanoparticles. If metal nanoparticles are much smaller than the wavelength of light and they can be approximately treated as spheres, optical properties of the dielectric-metal nanoparticle system may be described in terms of the refractive index $n$ of the dielectric, the frequency-dependent complex dielectric constant $\varepsilon(\omega)=\varepsilon_{1}(\omega)+i \varepsilon_{2}(\omega)$ of the metal particle, and the volume fraction of the metal $q$ by the MG theory

$$
\frac{\xi(\omega)-n^{2}}{\xi(\omega)+2 n^{2}}=q \frac{\varepsilon(\omega)-n^{2}}{\varepsilon(\omega)+2 n^{2}},
$$

where $\xi(\omega)=\xi_{1}(\omega)+i \xi_{2}(\omega)$ is the complex dielectric constant for the composite system, $i$ is the imaginary unit, and $q$ is the volume fraction of metal nanoparticles. Manipulating equation (1) and equating the real and imaginary parts, one gets

$$
\begin{aligned}
& \xi_{1}=n \frac{\left(\varepsilon_{1}^{2}+\varepsilon_{2}^{2}\right)(1+2 q)(1-q)+n^{2} \varepsilon_{1}\left(4 q^{2}+q+4\right)+2 n^{4}(1-q)(q+2)}{\left(\varepsilon_{1}^{2}+\varepsilon_{2}^{2}\right)(1-q)^{2}+2 n^{2} \varepsilon_{1}(1-q)(q+2)+n^{4}(q+2)^{2}} \\
& \xi_{2}=n^{2} \frac{9 n^{2} q \varepsilon_{2}}{\left(\varepsilon_{1}^{2}+\varepsilon_{2}^{2}\right)(1-q)^{2}+2 n^{2} \varepsilon_{1}(1-q)(q+2)+n^{4}(q+2)^{2}}
\end{aligned}
$$

In order to obtain the complete resonance frequency dependence on $q$, one needs to examine the optical properties of the system at a frequency of $\omega$. Let us assume that the dielectric constant of the metal nanoparticles can be described by the Drude theory as

$$
\begin{aligned}
& \varepsilon_{1}=1-\frac{\left(\omega_{p}^{2} \tau^{2}\right)}{1+\omega^{2} \tau^{2}}, \\
& \varepsilon_{2}=\frac{\omega_{p}^{2} \tau}{\omega\left(1+\omega^{2} \tau^{2}\right)},
\end{aligned}
$$

where $\omega_{p}=\left(4 \pi N e^{2} / m^{*}\right)$ is the plasma frequency of the free electrons. $e, N$, and $m^{*}$ are the element charge, the number density, and the effective mass of the conduction electrons, respectively.
2.1. Optical Conduction Resonance. Following the definition of conductivity, the conductivity of the system can be written as

$$
\begin{aligned}
S(\omega) & =\varepsilon_{0} \xi_{2} \omega \\
& =n^{2} \frac{9 n^{2} q \varepsilon_{2} \omega \varepsilon_{0}}{\left(\varepsilon_{1}^{2}+\varepsilon_{2}^{2}\right)(1-q)^{2}+2 n^{2} \varepsilon_{1}(1-q)(q+2)+n^{4}(q+2)^{2}},
\end{aligned}
$$

where $\varepsilon_{0}$ is the permittivity of vacuum. For the OCR frequency, one should find the maximum value of $S(\omega)$. Substituting $\varepsilon_{1}$ and $\varepsilon_{2}$ from equations (4) and (5) and taking

$$
\frac{\partial S(\omega)}{\partial \omega}=0
$$


we can find that one of the solutions is

$\omega_{c}=\left\{\frac{\omega_{p}^{2} \tau^{2}(2+q)-3-\left[9-6 \omega_{p}^{2} \tau^{2}(2+q)+9 q^{2} \omega_{p}^{4} \tau^{4}\right]^{1 / 2}}{6 \tau^{2}}\right\}^{1 / 2}$,

which corresponds to the OCR frequency [27, 28]. One can see this frequency depends on the metal particle volume fraction $q$. The computed results are shown in Figure 1. If we substitute $\omega_{c}$ from equation (8) into equation (2), after manipulating, one can get $\xi_{1}=0$. Since $\xi_{1}$ is related to the phase change of a propagating electromagnetic wave, this is an important factor to recognize the OCR phenomenon. This result will be used in the next sections.

2.2. Difference between $O C R$ and SPR. Since SPR coexists in metal nanoparticles, the OCR resulting absorption should not be confused with SPR and the resulting photon absorption. The two resonances always have different frequencies [27-32]. Only when $q$ is near zero, these two frequencies approach the same value. The surface energy loss function is defined as $L_{s}=-\operatorname{Im}(\xi+1)^{-1}[31,32]$. The SPR frequency $\omega_{s}$ is the frequency at which $L_{s}$ has the maximum value. Using equations (2) and (3), the condition creates the following equation,

$$
\begin{gathered}
n\left(\varepsilon_{1}^{2}+\varepsilon_{2}^{2}\right)(1+2 q)(1-q)+n^{3} \varepsilon_{1}\left(4 q^{2}+q+4\right) \\
+2 n^{5}(1-q)(q+2)+\left(\varepsilon_{1}^{2}+\varepsilon_{2}^{2}\right)(1-q)^{2} \\
+2 n^{2} \varepsilon_{1}(1-q)(q+2)+n^{4}(q+2)^{2}=0 .
\end{gathered}
$$

Substituting equations (4) and (5) into equation (9) for $\varepsilon_{1}$ and $\varepsilon_{2}$, one can find $\omega_{s}$ by numerically solving the equation. To compare with the computed OCR frequency, the computed SPR frequencies are also shown in Figure 1. Because $q$ ranges from 0 to $1, \omega_{s}$ falls between $3.5-6.5 \mathrm{eV}$ for a refractive index range of 1.3-2. Note that the OCR frequency is controllable from $4 \mathrm{eV}$ to lower than $1 \mathrm{eV}$. This offers a considerable opportunity to make photonic devices from UV to MIR. Surface plasmons may be excited by both photons and fast electrons, whereas the OCR can be excited by photons only.

2.3. Absorption Resonance at OCR Frequencies. The extinction coefficient $k$ of the system can be derived from the dielectric constant $\xi$.

$$
\eta=n_{r}+i k=\sqrt{\xi_{1}+i \xi_{2}}
$$

where $\eta$ is the complex refractive index, $n_{r}$ is the refractive index of the system, and $k$ is the extinction coefficient. From (10), one can obtain [33]

$$
k=\sqrt{-\frac{\xi_{1}}{2}+\frac{1}{2} \sqrt{\xi_{1}^{2}+\xi_{2}^{2}}}
$$

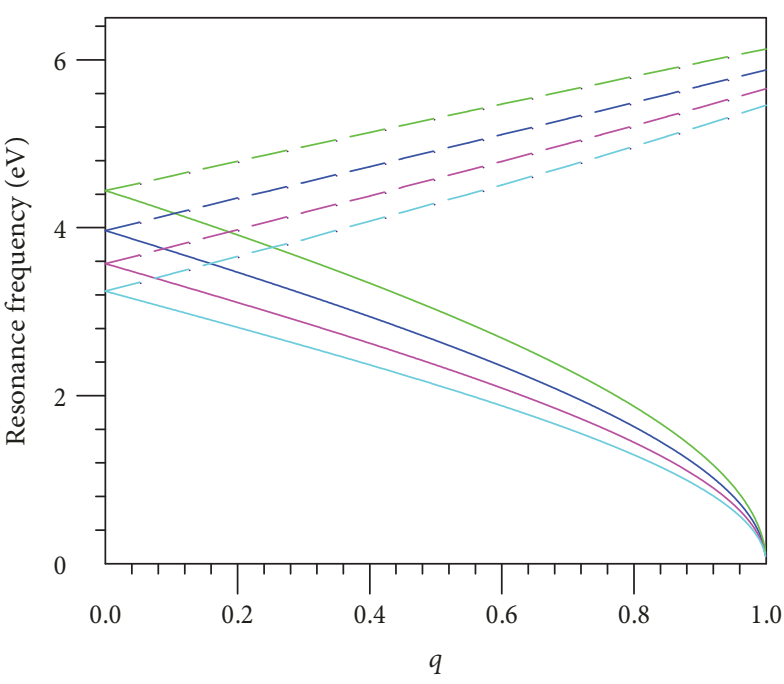

FIgURE 1: Computed OCR and SPR frequencies versus $q$ at different refractive indices of dielectric. The $\mathrm{Au}$ particle parameters of $\hbar \omega_{p}=9.3 \mathrm{eV}$ and $\tau=1.39 \times 10^{-13} \mathrm{~s}$ are used. Dashed lines are for SPR frequencies and solid lines correspond to the OCR frequencies. Refractive index values used are 1.3, 1.5, 1.7, and 1.9 (upper to lower). Note that two resonances occur at the same frequency only when $q$ is close to zero.

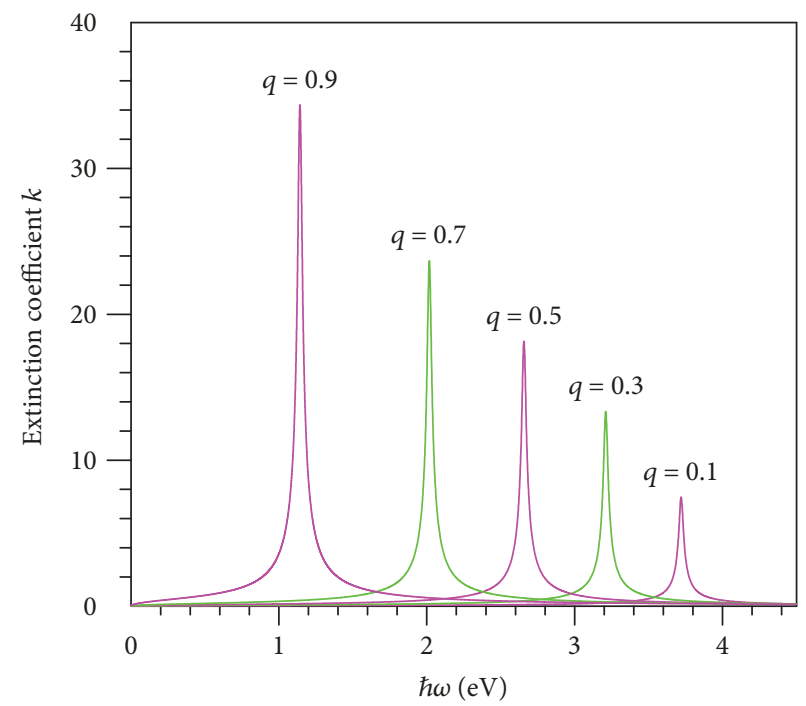

FIgURE 2: Computed resonance feature of extinction coefficient $k$ with different $q$ values. One can see that the resonance frequencies can be controlled ranging from $1 \mathrm{eV}$ to $4 \mathrm{eV}$ by varying $q$. The parameters are used as in Figure 1.

Since $\xi_{1}=0$ at the OCR resonance, one can readily compute $k$ using equations (3) and (10). Figure 2 shows the calculated results for different values of $q$ at $n=1.5$. One can see that absorption is significantly enhanced at the OCR frequencies.

2.4. Photoconductivity Feature at OCR Frequencies. Microscopically, when light is incident on the system, the free 
electrons in Au nanoparticles are driven by the periodic field. The free electrons respond to the periodic local field, oscillating within the volume of the nanoparticles. This is actually a periodic conduction current within the particles causing a periodic oscillation field around the particles-both periods being equal to that of the incident radiation. At a certain frequency the phases of the conduction currents in all the particles become nearly equal, resulting in a strong polarization field, which enhances the local field. Because the real part $\left(\xi_{1}\right)$ of the dielectric constant associates with the phase change while an electromagnetic wave propagates a distance, finally, at a frequency, $\xi_{1}=0$, where the local currents in all the metal particles are in phase with the driving electric field, the collective resonance of photoconductivity occurs. This resonance is not the normal free-electron plasmon resonance $[27,28]$. At the OCR frequencies, the photoconductivity can be calculated by using equation (6). The results for different resonance frequency at different $q$ are shown in Figure 3. One can see that the OCR peak of photoconductivity is higher with increasing $\mathrm{Au}$ nanoparticle volume fraction. For comparison, all values of the photoconductivity are normalized by the static conductivity $\left(\sigma_{0}=\varepsilon_{0} \omega_{p}{ }^{2} \tau\right)$ of the free electrons.

\section{Sample Preparation}

ESA is a recently developed technique to make thin films by alternate dipping, which was first used by Decher to fabricate polymer thin films [34]. Claus' group at Virginia Tech and NanoSonic improved this technique to be employed to make nanostructured dielectric, metal nanoparticle, and semiconductor quantum dot thin films [35-38]. The ESA process starts with a substrate that is cleaned and chemically functionalized with a solvent like hydrogen peroxide or sulfuric acid to effectively create a net uniform electric charge distribution on its surface. By alternate dipping of the substrate into the anionic and cationic molecular baths, a multilayered nanostructured thin film can gradually build up. There are several methods that can be used to positively or negatively functionalize gold and dielectric nanoparticles [38-42]. References [38, 39] report the methods to positively charge gold particles in gold colloids and Refs. [40-42] provide the techniques to negatively functionalized gold particles. In this work, poly-diallyl dimethyl ammonium chloride (PDDA) is used to positively functionalize gold particle colloids purchased from Sigma-Aldrich. Silica nanoparticles can be either positively or negatively functionalized by the methods reported in Refs. [43, 44]. Silica solutions were ordered from DuPont. Figure 4 briefly shows the ESA processes to fabricate 2D Au nanoparticle array-dielectric thin films. The value of $q$ can be controlled by dipping more metal nanoparticle monolayers or more dielectric monolayers. The detailed ESA processes can be found in Refs. [35-37].

The atomic force microscope (AFM) was used to image the surface of the samples for both the Au-PDDA and silica monolayers. The top view of the fabricated Au monolayer is shown in Figure 5 . One can see it clearly displays 2D

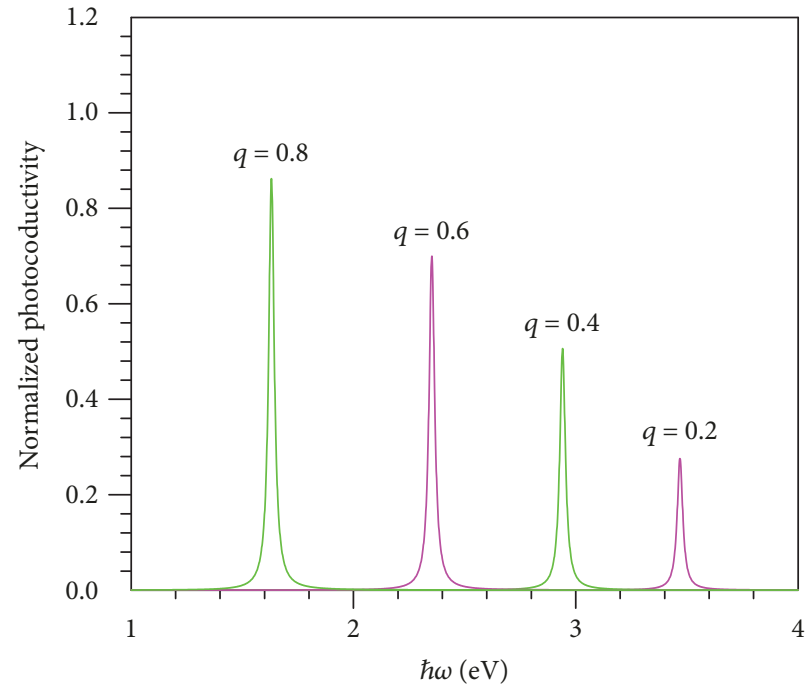

FIgURe 3: Computed resonance feature of photoconductivity $S$ according to equation (6). One can see that the resonance frequency can be controlled ranging from $1 \mathrm{eV}$ to $4 \mathrm{eV}$ by varying $q$ with $n=1.5$. When $q$ increases, the OCR frequency decreases. The parameters are used as in Figure 1.

nanostructured array with nanometer level interspacing between $\mathrm{Au}$ particles.

The surface of the silica monolayer was also pictured by the AFM technique. The image is shown in Figure 6. Au and $\mathrm{SiO}_{2}$ nanoparticles have an average diameter of $20 \mathrm{~nm}$ and $2 \mathrm{~nm}$, respectively. The gold nanoparticles were positively functionalized with PDDA $[38,39]$ and $\mathrm{SiO}_{2}$ nanoparticles were negatively charged $[43,44]$. The dipping process can be repeated to obtain a multilayer thin film with the thickness that is needed.

\section{Property Characterizations}

In order to find the OCR wavelength, the absorbance of these samples was measured. Figure 7 shows four absorption curves obtained from ESA gold nanoparticle/PDDA $/ \mathrm{SiO}_{2}$ thin films. One can see four different OCR peaks for four thin films with four different values of $q$. The OCR wavelength for the blue solid curve is $580 \mathrm{~nm}(q=0.66)$. The pink dasheddot curve shows an OCR wavelength of $700 \mathrm{~nm}(q=0.76)$. The rest two curves (solid-green and red dashed-dot) exhibit OCR wavelengths of $800 \mathrm{~nm}(q=0.81)$ and $1100 \mathrm{~nm}$ $(q=0.90)$, respectively.

The open-aperture $\mathrm{z}$-scan technique is used to investigate OCR-enhanced imaginary part of the third-order susceptibility. The setup is shown in Figure 8.

If nonlinear absorption is negligible, Beer's law [45] is commonly used to describe the linear relationship between absorbance and concentration of an absorbing species. However, if a sample exhibits strong nonlinear absorption, Beer's law needs to be modified to include the nonlinear absorption term. This work can be found in references [46-49]. Considering both linear and nonlinear absorption factors, when a laser beam propagates in a medium, the 


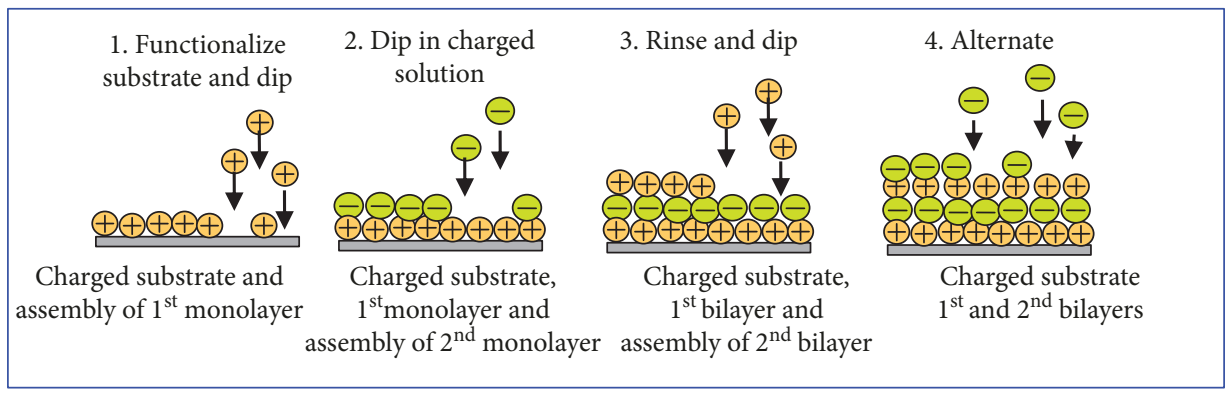

FIGURE 4: Schematic processes of fabricating Au and silica nanoparticle thin films. The Au and silica nanoparticles need to be functionalized before dipping $[34,38]$.

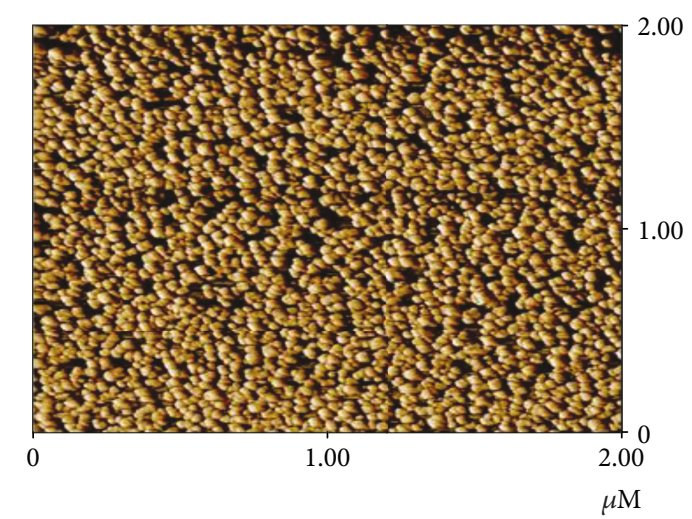

Figure 5: Top view of a monolayer of ESA Au particle-PDDA nanostructured array by AFM. The size of gold particles is nearly identical with an average diameter of $20 \mathrm{~nm}$.

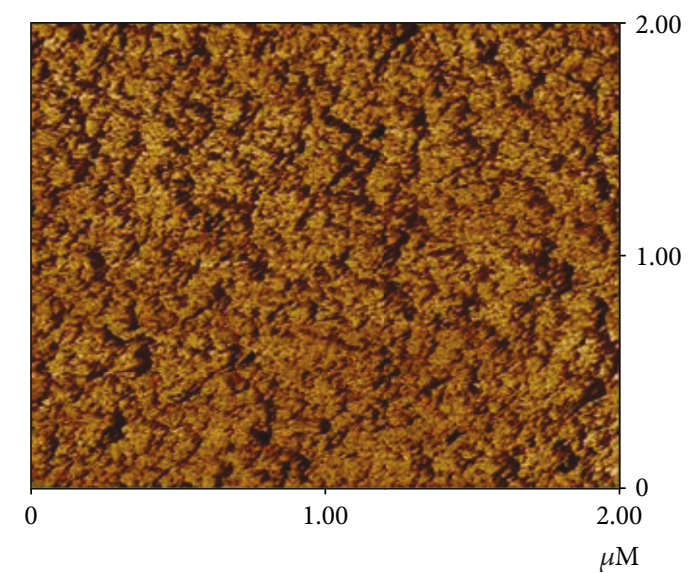

FIGURE 6: AFM image of the silica monolayer surface fabricated by ESA.

beam intensity changes along the propagation direction ( $z$ axis) can be approximately written as

$$
\frac{\partial I}{\partial z}=-\left(\alpha I+\beta I^{2}\right)
$$

where $\alpha$ is the linear absorption coefficient and $\beta$ is the nonlinear absorption (saturable, reverse saturable, or two-

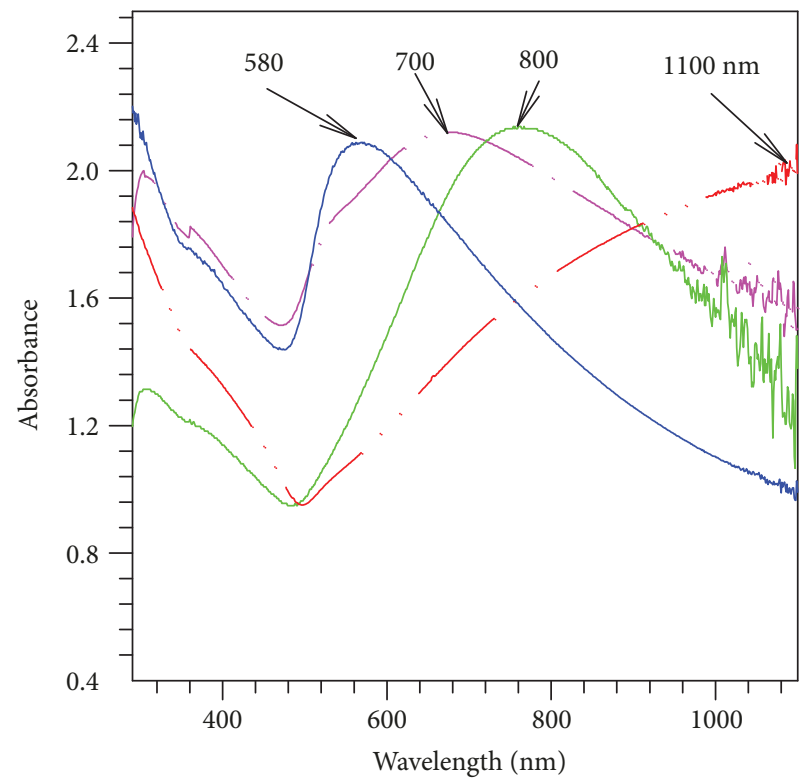

FIgURE 7: Absorption spectra for four different ESA films with four different values of $q$. The ESA films are Au nanoparticle-PDDA$\mathrm{SiO}_{2}$ thin films. One can see that the peaks range from visible to IR.

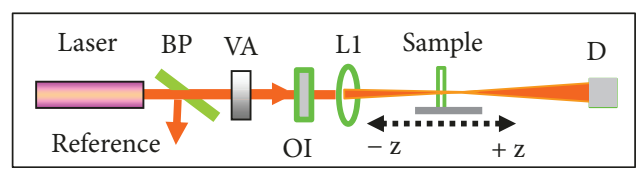

FIGURE 8: Setup of the open-aperture z-scan to measure nonlinear absorption. The sample is mounted on a translation stage and can be translated along the $z$ axis. BP: beamsplitter; OI: optical isolator; VA: variable attenuator; L: lens; D: photodetector.

photon absorptions) coefficient, which is related to the imaginary part of the third-order susceptibility $\chi^{(3)}$. If $\beta=0$, that means that only linear absorption (single-photon absorption) exists in the medium. If nonlinear absorption coexists in the medium, $\beta \neq 0$. Considering nonlinear absorption, the third-order nonlinear susceptibility is a complex quantity $[49,50]$

$$
\chi^{(3)}=\chi_{R}^{(3)}+i \chi_{I}^{(3)},
$$


where $\chi_{R}^{(3)}$ and $\chi_{I}^{(3)}$ correspond to the real and imaginary part of the third-order susceptibility, respectively. $\chi_{I}^{(3)}$ is related to the nonlinear absorption coefficient $\beta$ through $[45,46]$

$$
\chi_{I}^{(3)}=\frac{n_{r}^{2} \varepsilon_{0} c \lambda \beta}{2 \pi},
$$

where $n_{r}$ is the linear refractive index, $c$ is the speed of light, and $\lambda$ is the wavelength of the incident light. Using the value of $\beta$, one can obtain $\chi_{I}^{(3)}$.

Figure 8 shows the open-aperture z-scan setup $[49,50]$ which can be used to determine $\beta$ and $\chi_{I}{ }^{(3)}$. The open $\mathrm{z}$ scan is sensitive to nonlinear absorption. The normalized transmittance as a function of $z, T_{\text {Norm }}(z)$, is given by $[49,50]$

$$
T_{\text {Norm }}(z)=\sum_{m=0}^{\infty} \frac{\left(-\beta I_{0} L_{\text {eff }} /\left(1+z^{2} / z_{0}^{2}\right)\right)^{m}}{(m+1)^{3 / 2}},
$$

where $z_{0}$ is the Rayleigh diffraction length of the beam, $I_{0}$ is the on-axis peak intensity at the focus, $m$ is an integer, and $L_{\text {eff }}$ is the effective length of the sample. The laser beam is focused by the lens L1 and then travels through the sample. The position of the sample can be translated around the focal plane of the lens $\mathrm{L}_{1}$. When the sample is at the focus of L1, the laser intensity is greatest and nonlinear absorption will produce the greatest effect. If $\chi_{I}{ }^{(3)}>0$, the open $\mathrm{z}$-scan will result in a valley when the sample is near the focus, indicative of the OCR-induced absorption. If $\chi_{I}^{(3)}<0$, then the z-scan will produce a peak near the focus, indicating induced transparency (due to saturable absorption, for example). If one can obtain $T_{\text {Norm }}(z)$ experimentally, using equations (14) and (15), one can calculate the nonlinear absorption coefficient $\beta$ and $\chi_{I}{ }^{(3)}$.

The saturable absorption was measured for the sample with the OCR peak around $700 \mathrm{~nm}$ shown in Figure 7 (pink-dashed curve). In these measurements, a femtosecond laser system of Coherent Ti-sapphire Mira-900 that has a tunable range from $720 \mathrm{~nm}$ to $880 \mathrm{~nm}$ was used. The laser output has a pulse width of $200 \mathrm{fs}$ and a repetition rate of $76 \mathrm{MHz}$. Since the output power of the laser system at $720 \mathrm{~nm}$ is weak, $740 \mathrm{~nm}$ was used to conduct the measurement. The same measurement at $800 \mathrm{~nm}$ was also done for the purpose of comparison since $800 \mathrm{~nm}$ is further from the ORC peak than $740 \mathrm{~nm}$. The measured results at $740 \mathrm{~nm}(\bullet)$ and $800 \mathrm{~nm}$ are shown in Figure 9. For the two curves, the symbols indicate the measured results and the solid lines correspond to theoretical fitted results. The fits give $\chi_{I}^{(3)}=$ $-7.58 \times 10^{-10} \mathrm{esu}$ at $740 \mathrm{~nm}$ and $\chi_{I}^{(3)}=-4.03 \times 10^{-10}$ esu at $800 \mathrm{~nm}$. One can see the $\chi_{I}^{(3)}$ becomes smaller at $800 \mathrm{~nm}$ because it is further away from the OCR peak $(700 \mathrm{~nm})$, which indicates that the large $\chi_{I}^{(3)}$ may originate from OCR at around $700 \mathrm{~nm}$.

For comparison purpose, we measured nonlinear absorption in an Au nanoparticle colloid. The result is shown in Figure 10. The fit (solid-line) gives $\chi_{I}^{(3)}=-1.17 \times 10^{-14} \mathrm{esu}$. The colloid was purchased from Ted Pella Inc. with a number

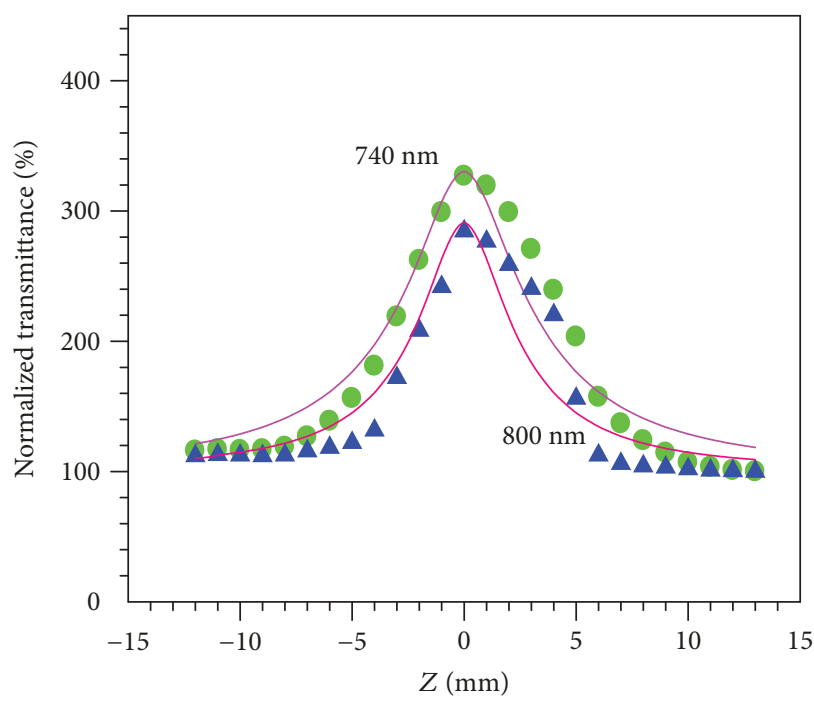

Figure 9: Measured saturable absorptions in an ESA Au nanoparticle-PDDA-SiO ${ }_{2}$ film at $740 \mathrm{~nm}$ (solid circles) and $800 \mathrm{~nm}$. The thicknesses of the films are about $250 \mathrm{~nm}$ calculated by monolayers. The power we used for these measurements is $100 \mathrm{~mW}$ before the sample. The laser beam has a diameter about $4 \mathrm{~mm}$ with a repetition rate of $76 \mathrm{MHz}$.

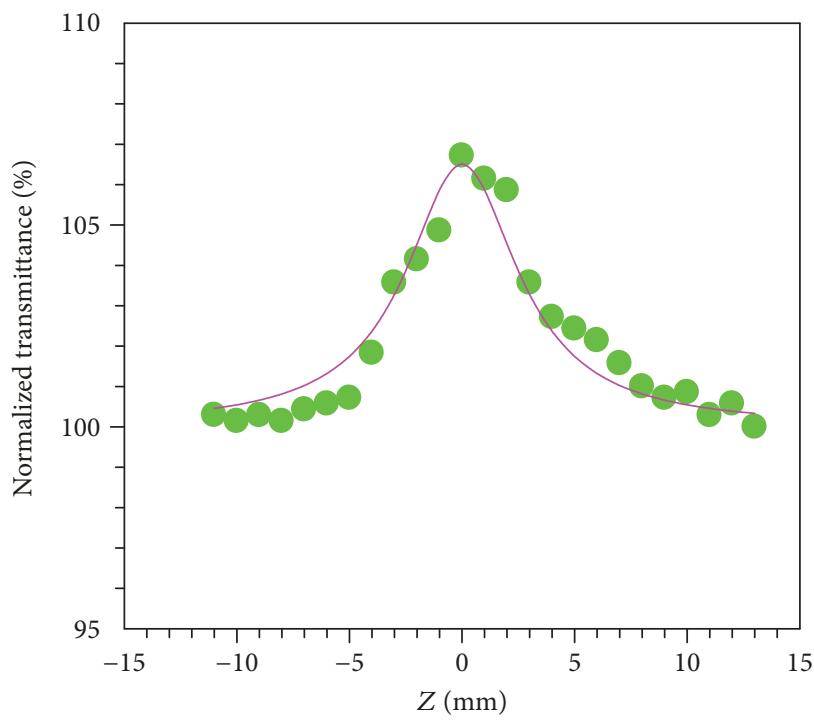

FIGURE 10: Measured nonlinear absorption in an Au particle colloid with a particle number density of $7 \times 10^{11} \mathrm{Au}$ particles $/ \mathrm{ml}$, which corresponds to $q=10^{-7}$.

density of $7 \times 10^{11} \mathrm{Au}$ particles $/ \mathrm{ml}$ and the particle has an average diameter of $20 \mathrm{~nm}$. Because one $20 \mathrm{~nm}$ Au particle has a size of $4.2 \times 10^{-24} \mathrm{~m}^{3}$, using the number density, one can get $\mathrm{q} \sim 10^{-7}$. Thus, the OCR effect is negligible in this colloid. Compared these data, one can see that $\chi_{I}^{\left({ }^{(3)}\right.}$ is enhanced about $10^{4}$ by OCR in the ESA film. The setup was calibrated by using carbon disulfide $\left(\mathrm{CS}_{2}\right)$ as a reference. At $800 \mathrm{~nm}$, the measured $\chi_{I}{ }^{(3)}$ for $\mathrm{CS}_{2}$ is $1.7 \times 10^{-14} \mathrm{esu}$, which is close to the reported value of $2 \times 10^{-14}$ esu [51]. 


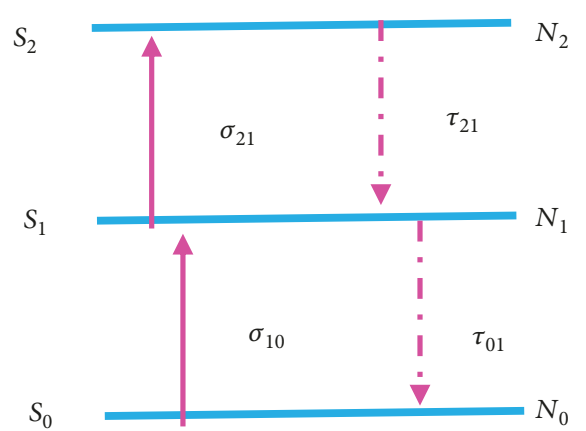

FIGURE 11: Energy-level diagram used to derive equation (16) from rate equations to explain the existence of RA in the ESA PDDA-Au thin films.

In order to briefly explain the saturable absorption (SA) we observed in the measurements, we consider the three electronic energy level model shown in Figure 11 that may exist in the polymer-Au composite films. $S_{i}$ is the energy level. $N_{i}$ stands for the electron population at the corresponding energy level. $\sigma_{i j}$ and $\tau_{i j}$ are the absorption cross section and lifetime between the two adjacent energy levels, respectively. Using the rate equations for the system $[46,52]$, the nonlinear absorption coefficient $\beta$ can be written,

$$
\beta=\frac{N_{1}}{N_{T}}(R-1),
$$

where $N_{T}=N_{1}+N_{2}$ and $R=\sigma_{21} / \sigma_{10}$. If $\sigma_{21}>\sigma_{10}, R>1$, the absorption is called the reverse saturable absorption (RSA). If $\sigma_{21}<\sigma_{10}, R<1$, the absorption is SA. Based on the measured data, the ESA PDDA-Au films have the property of SA. The detailed analysis and derivations may be found in Refs. [46, 52].

\section{Conclusions}

Au nanoparticles have strong nonlinear optical susceptibility; however, their optical properties are highly sensitive to interparticle distance $[24,53]$. In regular Au nanoparticle colloids, the particle volume fraction is very low and the interparticle distance can change dramatically. This may be used to interpret why $\mathrm{Au}$ colloids cannot show very large third-order optical susceptibility. Since the optical properties of Au nanoparticles are mainly governed by their interparticle distance, incorporating $\mathrm{Au}$ nanoparticles into a dielectric matrix to fabricate controllable superstructure of $\mathrm{Au}$ nanoparticles is crucial to developing photonic devices with large nonlinear optical susceptibility. In this paper, I have employed the suitable theory to compute the enhancement of third-order susceptibility $\chi^{(3)}$ from nanostructured thin films containing high volume fraction of Au nanoparticles. The nanostructured thin films were fabricated by the ESA technique that contain high volume fraction Au nanoparticles and the volume fraction can be controlled by dipping processes. The ESA thin films are characterized by using the open-aperture $\mathrm{z}$-scan technique. Based on the measured data, the imaginary part of third-order susceptibility has been extracted and been enhanced $\sim 10^{4}$ by OCR. The results reported in this paper could open up new avenues to develop high-volume metal nanoparticle-incorporated thin films with large thirdorder susceptibility.

\section{Data Availability}

The data used to support the findings of this study are included within the article.

\section{Conflicts of Interest}

The author declares that there is no conflict of interests regarding the publication of this paper.

\section{Acknowledgments}

The author would like to express his appreciation to Dr. Richard O. Claus and other researchers at NanoSonic Inc., Virginia, USA, for their assistance with this research work.

\section{References}

[1] N. L. Lepeshkin, A. Schweinsberg, G. Piredda, R. S. Bennink, and R. W. Boyd, "Enhanced nonlinear optical response of one-dimensional metal-dielectric photonic crystals," Physical Review Letters, vol. 93, no. 12, article 123902, 2004.

[2] A. K. Buin, P. F. de Chate, H. Nakotte, V. P. Drachev, and V. M. Shalaev, "Saturation effect in the optical response of Ag-nanoparticle fractal aggregates," Physical Review B, vol. 73, no. 3, article 035438, 2006.

[3] J. C. Maxwell-Garnett, "Colours in metal glasses and in metallic films," Philosophical Transactions of the Royal Society A: Mathematical, Physical and Engineering Sciences, vol. 203, no. 359-371, pp. 385-420, 1904.

[4] J. C. Maxwell-Garnet, "Colours in metal glasses, in metallic films, and in metallic solutions. II," Philosophical Transactions of the Royal Society A: Mathematical, Physical and Engineering Sciences, vol. 205, no. 387-401, pp. 237-288, 1906.

[5] G. Mie, "Beiträge zur optik trüber medien, speziell kolloidaler metallösungen," Annalen der Physik, vol. 330, no. 3, pp. 377445, 1908.

[6] D. Richard, P. H. Roussignol, and C. Flytzanis, "Surfacemediated enhancement of optical phase conjugation in metal colloids," Optics Letters, vol. 10, no. 10, pp. 511-513, 1985.

[7] M. J. Bloemer, J. W. Haus, and P. R. Ashley, "Degenerate fourwave mixing in colloidal gold as a function of particle size," Journal of the Optical Society of America B, vol. 7, no. 5, pp. 790-795, 1990.

[8] R. J. Gehr, G. L. Fischer, and R. W. Boyd, "Nonlinear-optical response of porous-glass-based composite materials," Journal of the Optical Society of America B, vol. 14, no. 9, pp. 23102314, 1997.

[9] X. C. Zeng, D. J. Bergman, P. M. Hui, and D. Stroud, "Effective-medium theory for weakly nonlinear composites," Physical Review B, vol. 38, no. 15, pp. 10970-10973, 1988.

[10] K. W. Yu, P. M. Hui, and D. Stroud, "Effective dielectric response of nonlinear composites," Physical Review B, vol. 47, no. 21, pp. 14150-14156, 1993. 
[11] J. E. Sipe and R. W. Boyd, "Nonlinear susceptibility of composite optical materials in the Maxwell Garnett model," Physical Review A, vol. 46, no. 3, pp. 1614-1629, 1992.

[12] F. Brouers, S. Blacher, A. N. Lagarkov, A. K. Sarychev, P. Gadenne, and V. M. Shalaev, "Theory of giant Raman scattering from semicontinuous metal films," Physical Review B, vol. 55, no. 19, pp. 13234-13245, 1997.

[13] H. Xu, J. Aizpurua, M. Kall, and P. Apell, "Electromagnetic contributions to single-molecule sensitivity in surfaceenhanced Raman scattering," Physical Review E, vol. 62, no. 3, pp. 4318-4324, 2000.

[14] D. A. Genov, A. K. Sarychev, V. M. Shalaev, and A. Wei, "Resonant field enhancements from metal nanoparticle arrays," Nano Letters, vol. 4, no. 1, pp. 153-158, 2004.

[15] E. Hao and G. C. Schatz, "Electromagnetic fields around silver nanoparticles and dimers," The Journal of Chemical Physics, vol. 120, no. 1, pp. 357-366, 2004.

[16] A. Gopinath, S. V. Boriskina, B. M. Reinhard, and L. D. Negro, "Deterministic aperiodic arrays of metal nanoparticles for surface-enhanced Raman scattering (SERS)," Optics Express, vol. 17, no. 5, pp. 3741-3753, 2009.

[17] G. Y. Panasyuk, J. C. Schotland, and V. A. Markel, "Classical theory of optical nonlinearity in conducting nanoparticles," Physical Review Letters, vol. 100, no. 4, article 047402, 2008.

[18] M. Chergui, A. Melikyan, and H. Minassian, "Calculation of surface plasmon frequencies of two, three, and four strongly interacting nanospheres," The Journal of Physical Chemistry C, vol. 113, no. 16, pp. 6463-6471, 2009.

[19] T. Yang and K. B. Crozier, "Surface plasmon coupling in periodic metallic nanoparticle structures: a semi-analytical model," Optics Express, vol. 16, no. 17, pp. 13070-13079, 2008.

[20] V. P. Drachev, A. K. Buin, H. Nakotte, and V. M. Shalaev, "Size dependent $\chi^{(3)}$ for conduction electrons in Ag nanoparticles," Nano Letters, vol. 4, no. 8, pp. 1535-1539, 2004.

[21] K. L. Kelly, E. Coronado, L. L. Zhao, and G. C. Schatz, "The optical properties of metal nanoparticles: the influence of size, shape, and dielectric environment," The Journal of Physical Chemistry B, vol. 107, no. 3, pp. 668-677, 2003.

[22] A. Ahmad and V. K. Tripathi, "Nonlinear absorption of femtosecond laser on a metal surface embedded by metallic nanoparticles," Applied Physics Letters, vol. 89, no. 15, article 153112, 2006.

[23] J. T. Seo, Q. Yang, W. J. Kim et al., "Optical nonlinearities of $\mathrm{Au}$ nanoparticles and Au/Ag coreshells," Optics Letters, vol. 34, no. 3, pp. 307-309, 2009.

[24] A. Mcleod, S. Kumar, K. C. Vernon, and K. Ostrikov, "Vertical graphene nanosheets coated with gold nanoparticles arrays: effects of interparticle spacing on optical response," Journal of Nanomaterials, vol. 2015, Article ID 230987, 7 pages, 2015.

[25] G. Jagannath, B. Eraiah, K. Naga Krishnakanth, and S. V. Rao, "Linear and nonlinear optical properties of gold nanoparticles doped borate glasses," Journal of Non-Crystalline Solids, vol. 482, pp. 160-169, 2018.

[26] F. Hache, D. Richard, and C. Flytzanis, "Optical nonlinearities of small metal particles: surface-mediated resonance and quantum size effects," Journal of the Optical Society of America, $B$, vol. 3, no. 12, pp. 1647-1655, 1986.

[27] J. P. Marton and J. R. Lemon, "Optical properties of aggregated metal systems, I. Theory," Physical Review B, vol. 4, no. 2, pp. 271-280, 1971.
[28] J. P. Marton and J. R. Lemon, "Optical properties of aggregated metal systems: real metals," Journal of Applied Physics, vol. 44, no. 9, pp. 3953-3959, 1973.

[29] M. Tajdidzadeh, A. B. Zakaria, Z. A. Talib, A. S. Gene, and S. Shirzadi, "Optical nonlinear properties of gold nanoparticles synthesized by laser ablation in polymer solution," Journal of Nanomaterials, vol. 2017, Article ID 4803843, 9 pages, 2017.

[30] M. H. Mezher, A. Nady, R. Penny, W. Y. Chong, and R. Zakaria, "Z-scan studies of the nonlinear optical properties of gold nanoparticles prepared by electron beam deposition," Applied Optics, vol. 54, no. 33, pp. 9703-9708, 2015.

[31] G. L. Tan, L. K. DeNoyer, R. H. French, M. J. Guittet, and M. Gautier-Soyer, "Kramers-Kronig transform for the surface energy loss function," Journal of Electron Spectroscopy and Related Phenomena, vol. 142, no. 2, pp. 97-103, 2004.

[32] A. Eguiluz and D. A. Campbell, "Surface energy-loss function for the inelastic scattering of electrons from a metal substrate with an overlayer of adsorbed alkali-metal atoms," Physical Review B, vol. 31, no. 12, pp. 7572-7579, 1985.

[33] U. Kreibig and M. Vollmer, "Optical properties of metal clusters," in Springer Series in Materials Science 25, pp. 2325, Springer, Berlin, 1995, chapter 2.

[34] G. Decher, "Fuzzy nanoassemblies: toward layered polymeric multicomposites," Science, vol. 277, no. 5330, pp. 12321237, 1997.

[35] L. Zhang, F. Zhang, K. Cooper, Y. Wang, Y. Liu, and R. O. Claus, "Electro-optic property measurements of electrostatically self-assembled ultrathin films," Optics Communications, vol. 186, no. 1-3, pp. 135-141, 2000.

[36] J. R. Heflin, C. Figura, D. Marciu, Y. Liu, and R. O. Claus, "Thickness dependence of second-harmonic generation in thin films fabricated from ionically self-assembled monolayers," Applied Physics Letters, vol. 74, no. 4, pp. 495-497, 1999.

[37] L. Zhang, F. Zhang, Y. Wang, and R. Claus, "Linear electrooptic tensor ratio determination and quadratic electro-optic modulation of electrostatically self-assembled CdSe quantum dot films," The Journal of Chemical Physics, vol. 116, no. 14, pp. 6297-6304, 2002.

[38] Y. Liu and R. O. Claus, "Strong enhancement of optical absorbance from ionic self-assembled multilayer thin films of nanocluster Pt and polymer dye," Journal of Applied Physics, vol. 85, no. 1, pp. 419-424, 1999.

[39] Y. Liu, Y. Wang, and R. O. Claus, "Layer-by-layer ionic selfassembly of Au colloids into multilayer thin-films with bulk metal conductivity," Chemical Physics Letters, vol. 298, no. 4-6, pp. 315-319, 1998.

[40] G. H. Woehrle, M. G. Warmer, and J. E. Hutchison, "Ligand exchange reactions yield subnanometer, thiol-stabilized gold particles with defined optical transitions," The Journal of Physical Chemistry B, vol. 106, no. 39, pp. 9979-9981, 2002.

[41] P. Kannan and S. A. John, "Synthesis of mercaptothiadiazolefunctionalized gold nanoparticles and their self-assembly on $\mathrm{Au}$ substrates," Nanotechnology, vol. 19, no. 8, article 085602, 2008.

[42] C. K. Lee, R. Jordan, A. Ulman et al., "Novel one-phase synthesis of thiol-functionalized gold, palladium, and iridium nanoparticles using superhydride," Langmuir, vol. 15, no. 10, pp. 3486-3491, 1999.

[43] A. Y. Kim and J. C. Berg, "Fractal heteroaggregation of oppositely charged colloids," Journal of Colloid and Interface Science, vol. 229, no. 2, pp. 607-614, 2000. 
[44] H. Shibata, M. Sato, S. Watanabe, and M. Matsumoto, "Selfassembled arrays of silica particles on the patterns reflecting the phase-separated structures of mixed Langmuir-Blodgett films," Colloids and Surfaces A: Physicochemical and Engineering Aspects, vol. 346, no. 1-3, pp. 58-60, 2009.

[45] R. V. Maikala, "Modified Beer's law - historical perspectives and relevance in near-infrared monitoring of optical properties of human tissue," International Journal of Industrial Ergonomics, vol. 40, no. 2, pp. 125-134, 2010.

[46] S. Hughes and B. Wherrett, "Multilevel rate-equation analysis to explain the recent observations of limitations to optical limiting dyes," Physical Review A, vol. 54, no. 4, pp. 35463552, 1996.

[47] G. Sreekumar, P. G. Louis Frobel, C. I. Muneera, K. Sathiyamoorthy, C. Vijayan, and C. Mukherjee, "Saturable and reverse saturable absorption and nonlinear refraction in nanoclustered amido black dye-polymer films under low power continuous wave $\mathrm{He}-\mathrm{Ne}$ laser light excitation," Journal of Optics A: Pure and Applied Optics, vol. 11, no. 12, article 125204, 2009.

[48] G. S. He, L. Yuan, Y. Cui, M. Li, and P. N. Prasad, "Studies of two-photon pumped frequency-upconverted lasing properties of a new dye material," Journal of Applied Physics, vol. 81, no. 6, pp. 2529-2537, 1997.

[49] M. S. Bahae, A. A. Said, T. H. Wei, D. J. Hagan, and E. W. V. Stryland, "Sensitive measurement of optical nonlinearities using a single beam," IEEE Journal of Quantum Electronics, vol. 26, no. 4, pp. 760-769, 1990.

[50] D. D. Smith, G. Fischer, R. W. Boyd, and D. A. Gregory, "Cancellation of photoinduced absorption in metal nanoparticle composites through a counterintuitive consequence of local field effects," Journal of the Optical Society of America B, vol. 14, no. 7, pp. 1625-1631, 1997.

[51] R. A. Ganeev, A. I. Ryasnyansky, N. Ishizawa et al., "Two- and three-photon absorption in $\mathrm{CS}_{2}$," Optics Communications, vol. 231, no. 1-6, pp. 431-436, 2004.

[52] X. Deng, X. Zhang, Y. Wang, Y. Song, S. Liu, and C. Li, "Intensity threshold in the conversion from reverse saturable absorption to saturable absorption and its application in optical limiting," Optics Communications, vol. 168, no. 1-4, pp. 207-212, 1999.

[53] C. P. Byers, H. Zhang, D. F. Swearer et al., "From tunable coreshell nanoparticles to plasmonic drawbridges: active control of nanoparticle optical properties," Science Advances, vol. 1, no. 11, article e1500988, 2015. 


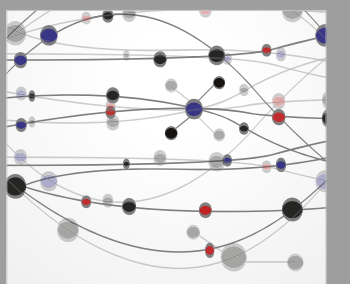

The Scientific World Journal
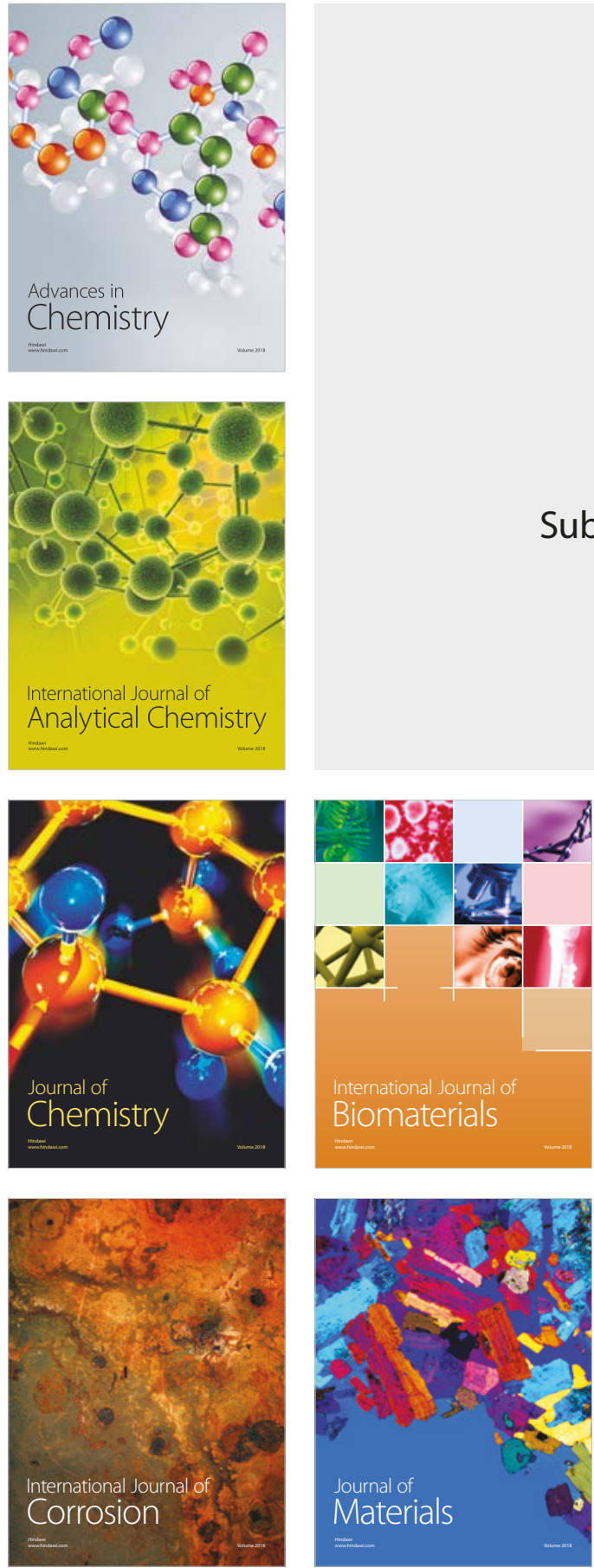

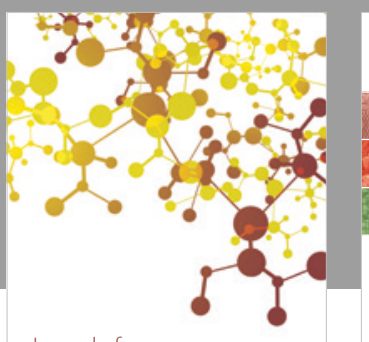

Journal of

Applied Chemistry
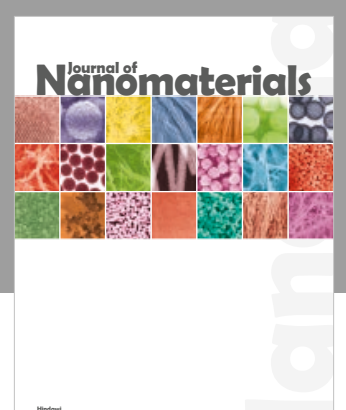

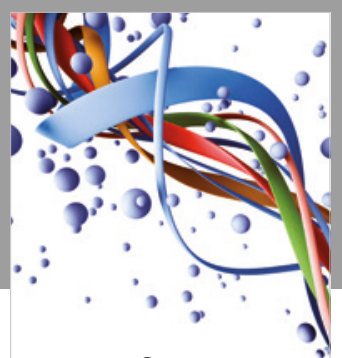

Scientifica

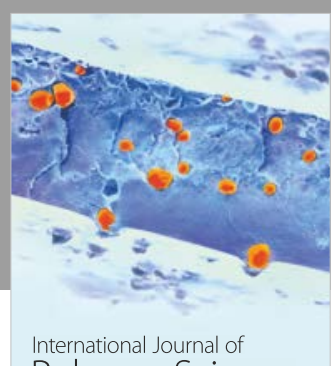

Polymer Science

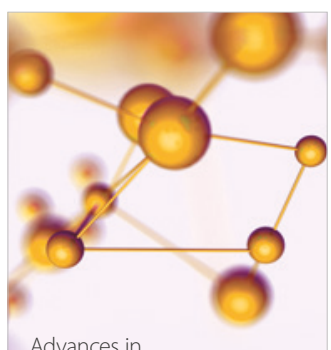

Physical Chemistry
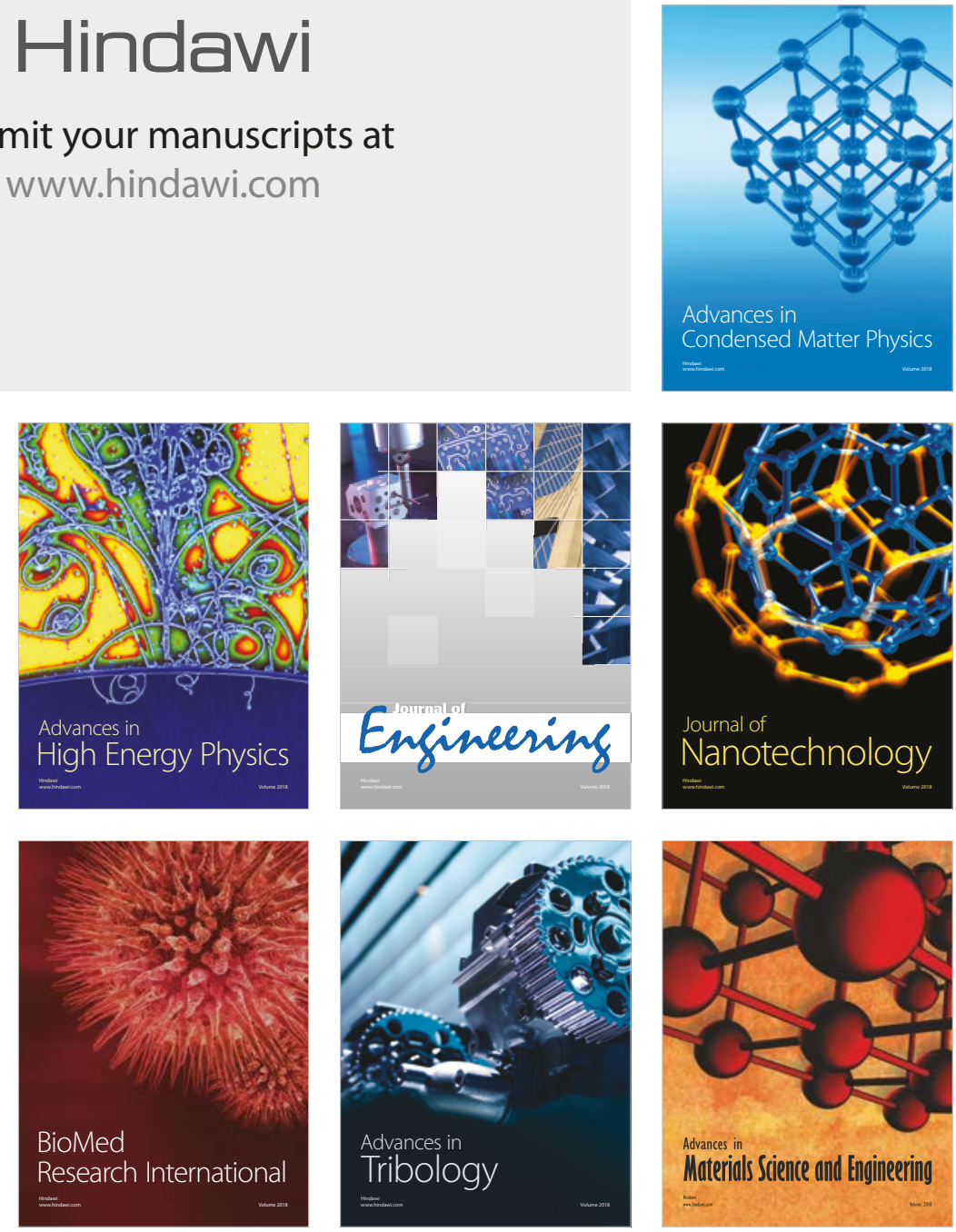